\title{
Phase III trial of mipomersen in heterozygous FH
}

Various studies have shown that LDLcholesterol and apolipoprotein B levels are reduced with mipomersen use in individuals with homozygous familial hypercholesterolemia (FH). However, many clinicians, including several on an FDA panel assessing the drug for use in patients with homozygous $\mathrm{FH}$, have expressed concerns about the adverse effects that mipomersen has on the liver. These effects include elevations in hepatic transaminase levels and in hepatic fat. Nevertheless, on 18 October 2012, an FDA panel voted 9-6 in favor of recommending that the FDA approve mipomersen for use as an add-on therapy in patients with homozygous $\mathrm{FH}$ who are already on other lipid-lowering therapy and on a low-fat diet.

For the setting of heterozygous $\mathrm{FH}$ and $\mathrm{CAD}$, a new phase III trial has compared mipomersen ( $200 \mathrm{mg}$ weekly by selfadministered subcutaneous injection) with placebo in 124 patients who were already taking maximally tolerated doses of statin. The primary efficacy end point was change in LDL-cholesterol level from baseline at 28 weeks (or 2 weeks after last dose of mipomersen or placebo in those who did not complete the study). Safety was assessed via various outcome measures.

Levels of LDL cholesterol were reduced in patients assigned mipomersen, but not in those assigned placebo $(-28.0 \%$ vs $+5.2 \%, P<0.001)$. Almost half of patients (45.1\%) assigned mipomersen, but only $4.9 \%$ of those assigned placebo, achieved the target LDL-cholesterol level of $<2.6 \mathrm{mmol} / 1$ ( $100 \mathrm{mg} / \mathrm{dl})$. The mipomersen-associated reduction in LDLcholesterol level was greater in females $(-40.6 \%)$ than in males $(-20.0 \%)$.

Notably, however, $10.8 \%$ of patients receiving mipomersen, but none of the patients in the placebo group, withdrew from treatment during the trial. Injectionsite reactions occurred about twice as often in patients receiving mipomersen and change in hepatic fat content was significantly greater in the mipomersen group $(4.9 \%$ vs $0.4 \%, P<0.001)$. Moreover, alanine-aminotransferase elevations greater than three times the upper limit of normal occurred in $14.5 \%$ of individuals on mipomersen versus $2.4 \%$ on placebo. These elevations were confirmed $\geq 1$ week later in $6 \%$ and in none of the patients assigned to mipomersen and placebo, respectively.

The authors of the report highlight that the "clinical implications of such increases in hepatic fat, and transaminase elevations, and the overall safety ... must be elucidated in longer-term studies". They also point out that future studies are needed to determine whether titration to lower doses of mipomersen would be safer, but associated with preserved efficacy.

Bryony M. Mearns

Original article Stein, E. A. et al. Apolipoprotein B synthesis
inhibition with mipomersen in heterozygous familial
hypercholesterolemia: results of a randomized, double-
blind, placebo controlled trial to assess efficacy and safety
as add-on therapy in patients with coronary artery disease.
Circulation doi:10.1161/CIRCULATIONAHA.112.104125

